Supporting Information

\title{
Multiplexed Centrifugal Microfluidic System for Dynamic Solid-Phase Purification of Polynucleic Acids Direct from Buccal Swabs
}

\author{
Leah M. Dignan ${ }^{1 *}$, M. Shane Woolf ${ }^{1}$, Christopher J. Tomley ${ }^{1}$, Aeren Q. Nauman ${ }^{2}$, and James P. Landers ${ }^{1,3,4}$ \\ ${ }^{1}$ Department of Chemistry, University of Virginia, Charlottesville, VA 22904, USA \\ ${ }^{2}$ TeGrex Technologies, Charlottesville, VA 22903, USA \\ ${ }^{3}$ Department of Mechanical and Aerospace Engineering, University of Virginia Charlottesville, VA 22904, \\ USA \\ ${ }^{4}$ Department of Pathology, University of Virginia Charlottesville, VA 22904, USA
}

Corresponding Author: Imd4bt@virginia.edu 


\section{$\underline{\text { Table of Content }}$}

Fig. S1. Images of the integrated fluidic control protocol for on-disc NA preparation.

Fig. S2. Flow chart depicting stepwise on-disc flow control protocol.

Fig. S3. Optimization of extraction chamber geometry.

Fig. S4. Objective visual determination of the volume of metered lysate.

Fig. S5. Evaluation of intermediate TE wash necessity.

Fig. S6. Tartrazine standard curve used in dye-based determination of eluate purity.

Video S1. Back-and-forth bead sweeping through the extraction chamber. 


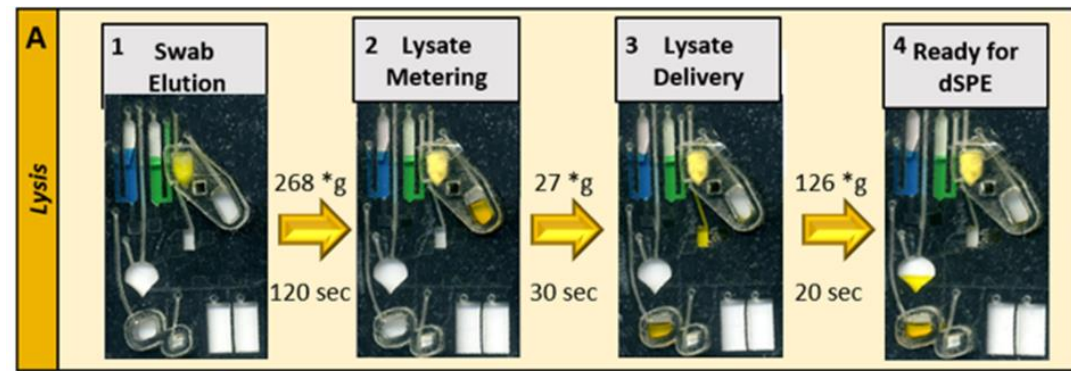

$\square_{\text {Isopropanol }} \square_{1 X T E} \quad \square$ Cellular Lysate
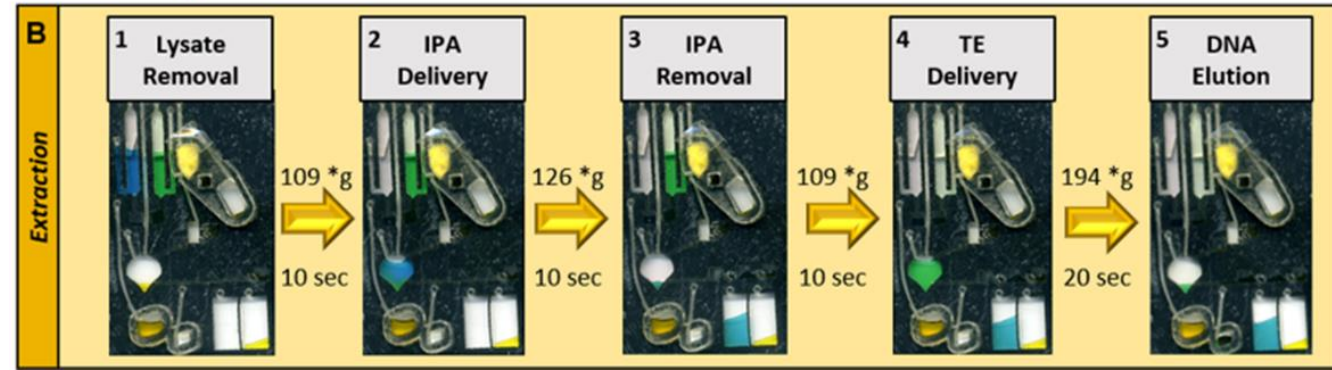

Figure S1. Images of the integrated fluidic control protocol for on-disc NA preparation. By leveraging the valving protocol described in Figure 2, three aqueous dyes were used to represent the lysate (yellow), isopropanol (blue), and TE elution buffer (green) to visually depict the fluidic protocol used for on-disc dSPE. (A) After incubation, the resulting lysate (yellow) was centrifugally eluted from the swab cutting, and (2) collected in the swab elution chamber. (3) A 2 uL aliquot of lysate was subsequently metered out, with the excess driven to the lysate overflow chamber. (4) The 2 uL lysate aliquot was delivered to the extraction chamber where purification could then proceed using reagents preloaded reagents. (B) After NA capture, (1) the supernatant lysate was driven to waste and (2) isopropanol was delivered to the extraction chamber (3) After washing the beads, the supernatant isopropanol was spun out of the extraction chamber. (4) Finally, the TE elution buffer was introduced to the extraction chamber to facilitate NA elution from the beads and, (5) the purified NA solution was transferred to the NA recovery chamber. 


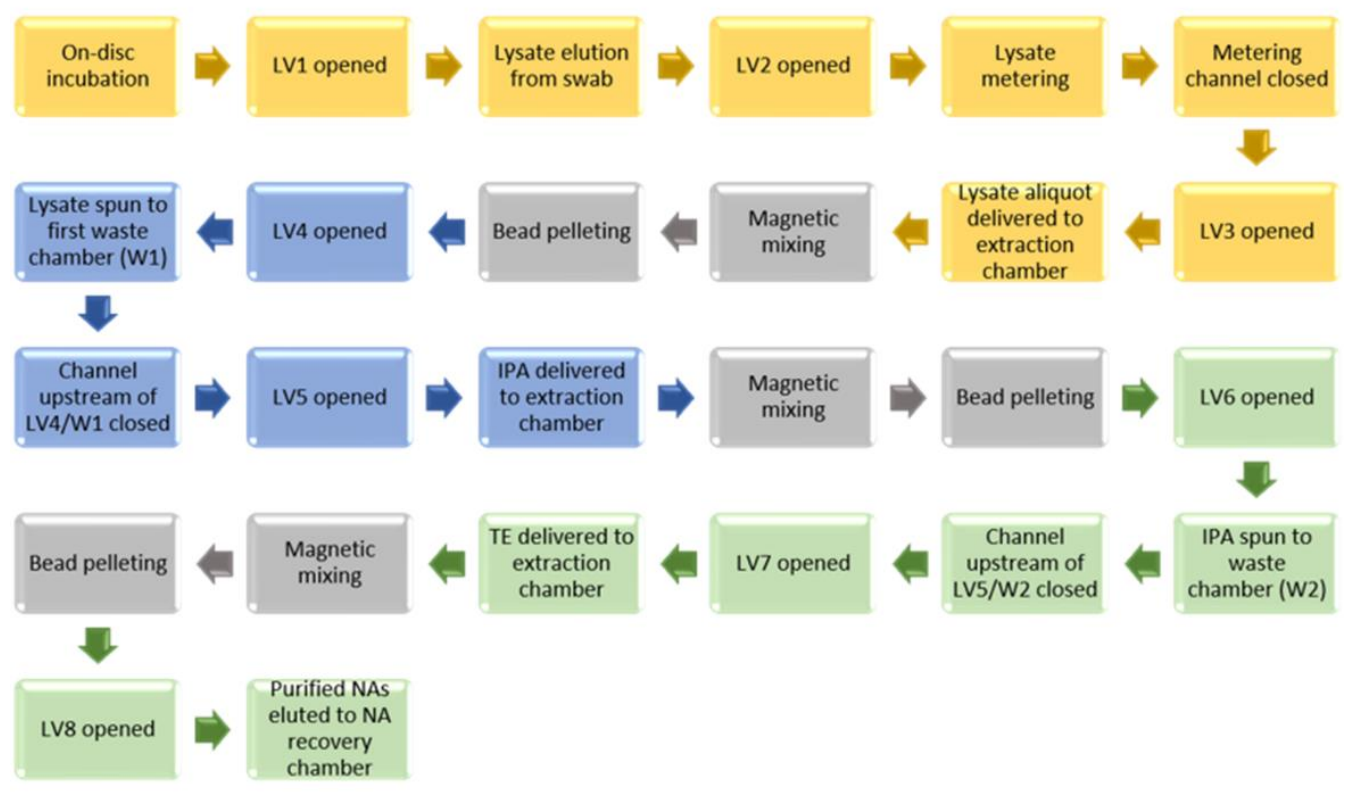

Figure S2. Flow chart depicting stepwise on-disc flow control protocol. This includes valving and fluidic events to enable microfluidic lysis (yellow), the isopropanol wash (blue), and the TE wash (green). Bead manipulation steps are shown in grey.
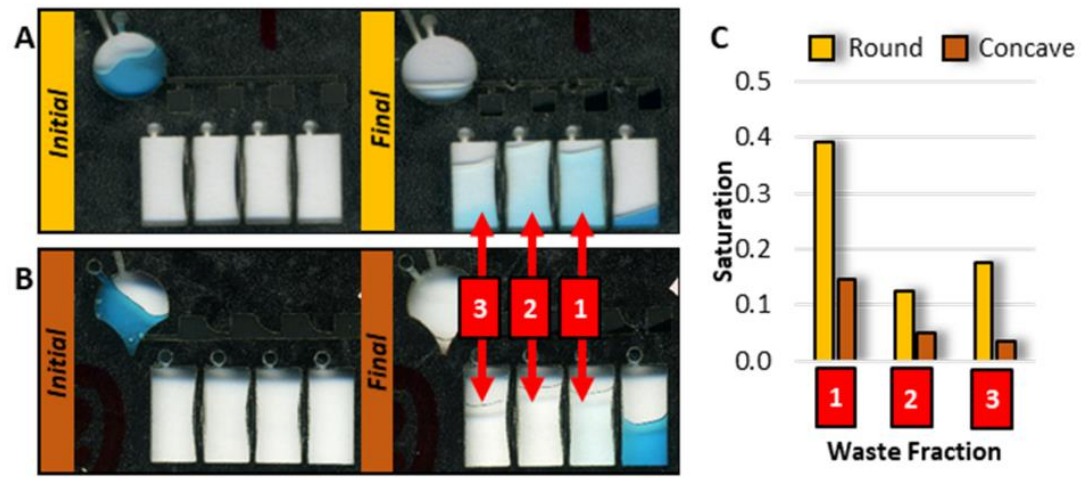

Figure S3. Optimization of extraction chamber geometry. Empirical image analysis (saturation) was used to characterize removal of blue dye, used to visually represent the lysate, from a (A) round and (B) concave pointed extraction chamber during on-disc dSPE. It was visually apparent that all three fractions (1-3) eluted from the round extraction chamber contained blue dye, whereas fractions 2-3 downstream of the concave pointed chamber appear colorless. (C) Using a round chamber shape, all waste fractions exhibited elevated saturation values relative to pure water (0.057), whereas utilizing a concave pointed chamber resulted in return to baseline saturation by the second wash (saturation $=0.050$ ). 


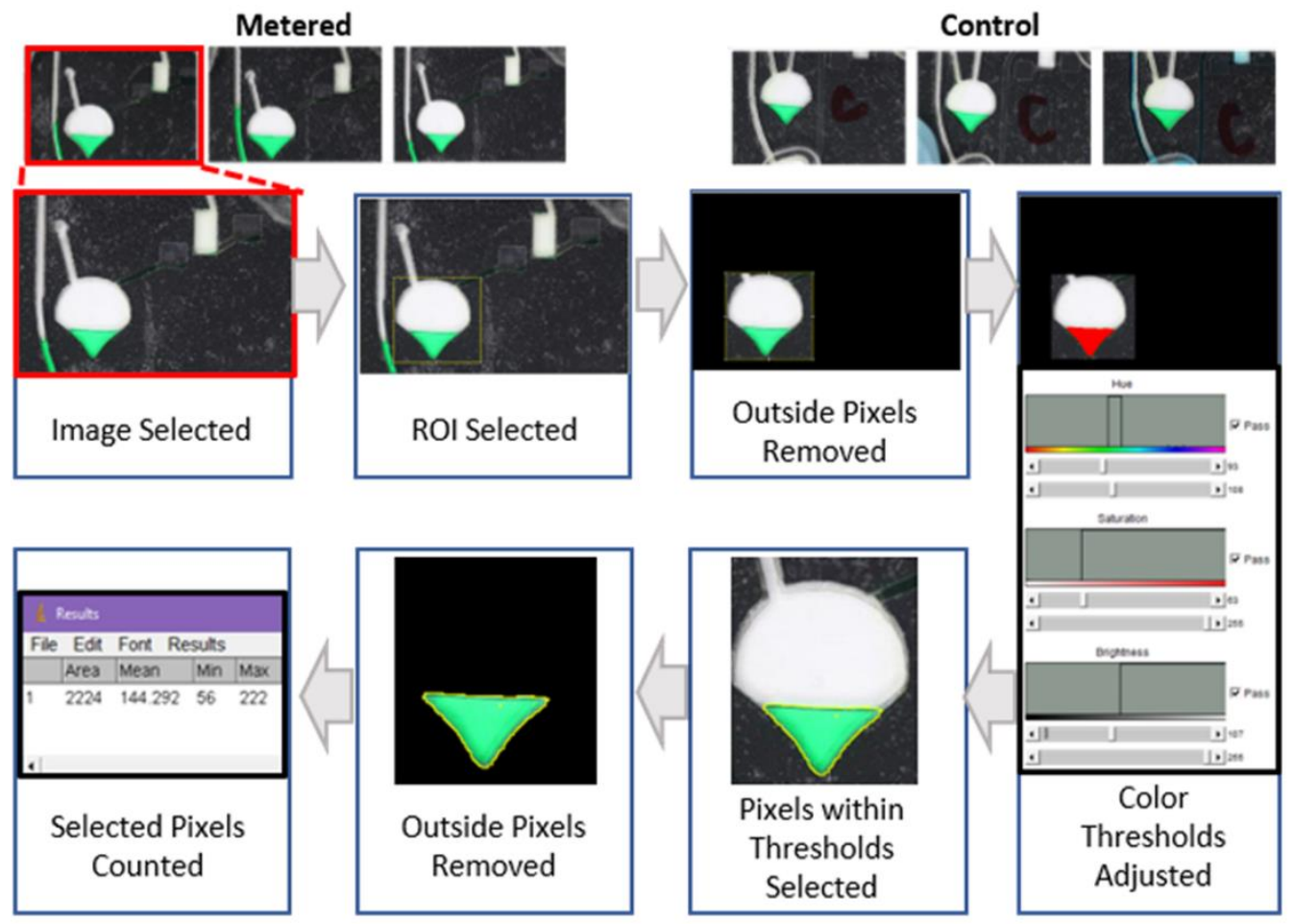

Figure S4. Objective visual determination of the volume of metered lysate. Using an aqueous green dye ( $28 \mu \mathrm{L})$ to visually represent the cellular lysate, we executed the on-disc spin and valving protocol for lysis. To measure the volume of the dye aliquot delivered to the extraction chamber, a surrounding rectangular region of interest was selected and all pixels outside of these bounds were cleared (ImageJ). By adjusting the image color thresholds, only the pixels associated with the green dye were selected and counted. The same color threshold adjustment values were applied uniformly across images.

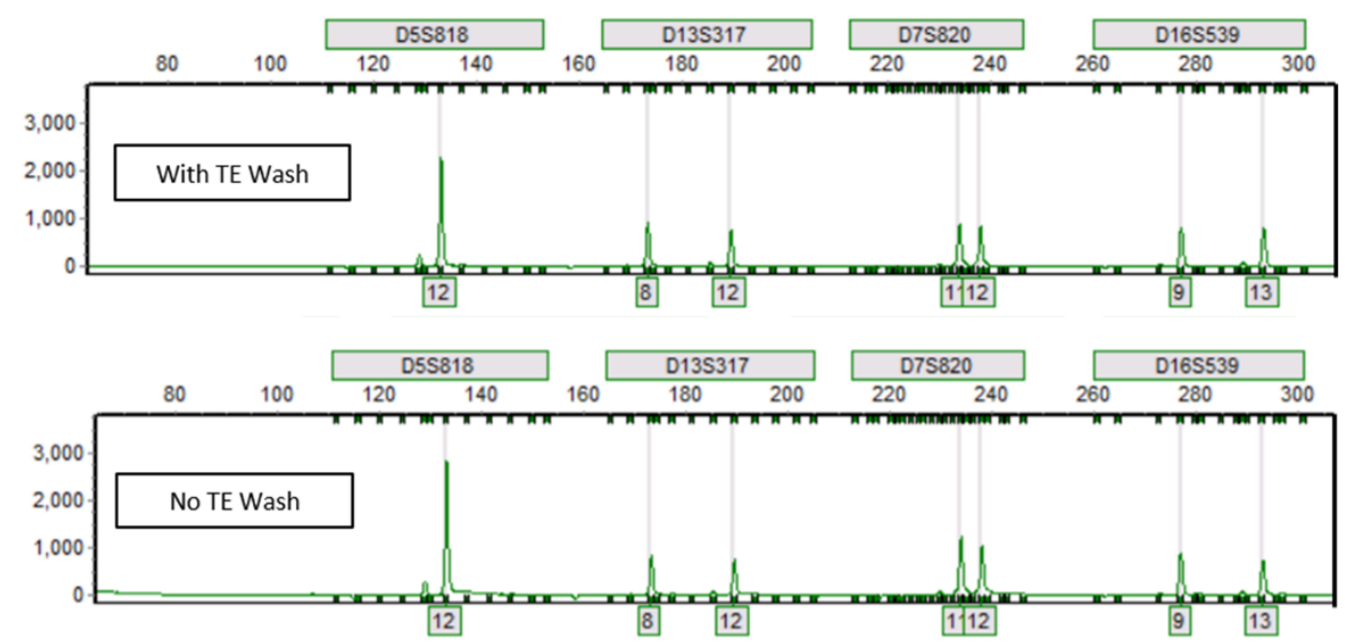

Figure S5. Evaluation of intermediate TE wash necessity. STR profiles from DNA obtained via in-tube dSPE with and without the intermediate TE wash. Peak heights were higher without the intermediate TE wash, and no signs of inhibition were observed, proving the intermediate wash to be unnecessary. 


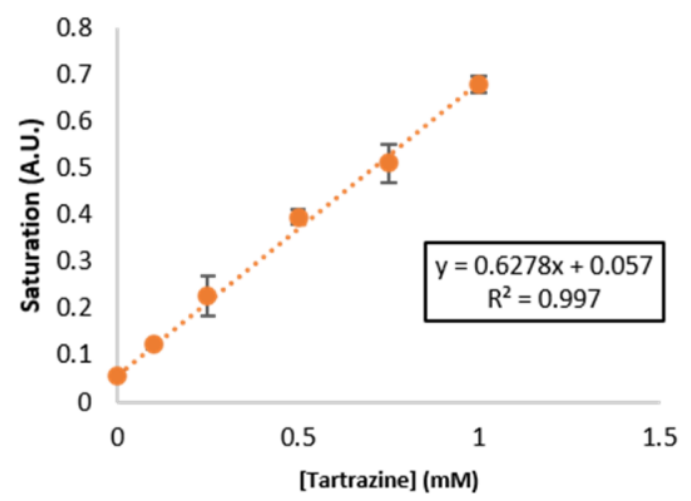

Figure S6. Tartrazine standard curve used in dye-based determination of eluate purity. Calibration curve constructed by measuring the saturation of aqueous tartrazine dilutions loaded into microfluidic chambers. Each point represents the mean of triplicate measurements with error bars depicted as one standard deviation in both directions. Tartrazine concentration (mM) and saturation (A.U.) exhibited an excellent linear correlation $\left(R^{2}=0.997\right)$.

Video S1. Back-and-forth bead sweeping through the extraction chamber. Bead movement was driven by bidirectional rotating magnetic field application (approximately $4 \mathrm{X}$ acceleration relative to real-time). 